\title{
Review
}

María Pérez-Burgos and Lotte Søgaard-Andersen*

\section{Biosynthesis and function of cell-surface polysaccharides in the social bacterium Myxococcus xanthus}

https://doi.org/10.1515/hsz-2020-0217

Received June 16, 2020; accepted August 4, 2020; published online August 24, 2020

\begin{abstract}
In bacteria, cell-surface polysaccharides fulfill important physiological functions, including interactions with the environment and other cells as well as protection from diverse stresses. The Gram-negative delta-proteobacterium Myxococcus xanthus is a model to study social behaviors in bacteria. M. xanthus synthesizes four cellsurface polysaccharides, i.e., exopolysaccharide (EPS), biosurfactant polysaccharide (BPS), spore coat polysaccharide, and $\mathrm{O}$-antigen. Here, we describe recent progress in elucidating the three Wzx/Wzy-dependent pathways for EPS, BPS and spore coat polysaccharide biosynthesis and the $\mathrm{ABC}$ transporter-dependent pathway for O-antigen biosynthesis. Moreover, we describe the functions of these four cell-surface polysaccharides in the social life cycle of $M$. xanthus.
\end{abstract}

Keywords: biosurfactant polysaccharide; exopolysaccharide; $\mathrm{O}$-antigen; spore coat polysaccharide; sporulation; type IV pili.

\section{Introduction: polysaccharides and the bacterial cell envelope}

In bacteria, the cell envelope serves as the important first barrier of protection against the environment, confers resistance to internal turgor pressure, and is also an important site of interaction of bacterial cells with the environment as well as with eukaryotic cells and other

*Corresponding author: Lotte Søgaard-Andersen, Department of Ecophysiology, Max Planck Institute for Terrestrial Microbiology, Karlvon-Frisch Str. 10, D-35043 Marburg, Germany, e-mail: sogaard@mpimarburg.mpg.de. https://orcid.org/0000-0002-0674-0013

María Pérez-Burgos, Department of Ecophysiology, Max Planck Institute for Terrestrial Microbiology, Karl-von-Frisch Str. 10, D-35043 Marburg, Germany bacteria (Silhavy et al. 2010). Accordingly, antibacterial compounds often target the synthesis of cell envelope components (Raetz and Whitfield 2002; Sperandeo et al. 2019). In Gram-positive bacteria, the cell envelope consists of a single membrane composed of phospholipids surrounded by a thick peptidoglycan (PG) layer and also contains wall teichoic acids and lipoteichoic acids covalently coupled to the PG and membrane lipids, respectively. By contrast, Gram-negative bacteria possess an inner membrane (IM) composed of phospholipids (Bogdanov et al. 2020), a thinner PG layer and an asymmetric outer membrane (OM) in which the inner leaflet is composed of phospholipids and the outer leaflet mainly of lipopolysaccharide (LPS) (Silhavy et al. 2010; Whitfield and Trent 2014). LPS molecules encompass three regions, the hydrophobic lipid A, a core oligosaccharide that is attached to lipid $\mathrm{A}$, and the highly variable O-antigen polysaccharide that is attached to the core and is composed of repeating oligosaccharide units (Whitfield and Trent 2014). In addition, bacterial cells are often covered by a layer of secreted polysaccharides. These can be capsular polysaccharides, which are covalently coupled to components in the cell envelope, or polysaccharides that are only loosely associated with the envelope. From here on, we refer to O-antigen and secreted polysaccharides as cellsurface polysaccharides.

In Gram-negative bacteria, cell-surface polysaccharides not only have important protective functions, e.g., protection from desiccation stress, predation and the immune system (Silhavy et al. 2010) but are also important virulence factors (Raetz and Whitfield 2002; Whitfield 2006). Moreover, secreted polysaccharides constitute an important component of the extracellular matrix in biofilms (Flemming et al. 2016). Cell-surface polysaccharides can also have more specialized functions in individual species, including: (i) stimulation of the symbiosis between rhizobia and legume plants by promoting adhesion of bacterial cells to root hairs (Downie 2010), (ii) contributing to swarming of bacteria on surfaces by lowering friction (Partridge and Harshey 2013), (iii) formation of the cyst coat 
in Azotobacter vinelandii to generate the desiccation resistant cyst cells (Sabra and Zeng 2009), and the spore coat in Myxococcus xanthus to generate the environmentally resistant spores (Holkenbrink et al. 2014), and (iv) acting as a surfactant to reduce surface tension whereby spore aggregation in several Bacillus spp. is lowered ( $\mathrm{Li}$ et al. 2016).

The Gram-negative delta-proteobacterium $M$. xanthus is a model organism to study social behaviors in bacteria (Konovalova et al. 2010). M. xanthus synthesizes four different cell-surface polysaccharides that have important functions in its the social lifestyle. Here, we describe recent progress in elucidating the involved biosynthetic pathways and the functions of these polysaccharides in the social lifestyle of $M$. xanthus.

\section{Cell-surface polysaccharide biosynthesis pathways in bacteria: an overview}

Synthesis of cell-surface polysaccharides in bacteria typically occurs via one of three different assembly schemes: the Wzx/Wzy-, the ABC transporter- and the synthasedependent pathways (Figure 1) (Whitfield et al. 2020). In the Wzx/Wzy- and ABC transporter-dependent pathways, synthesis starts with the transfer of a sugar-1-phosphate (sugar-1-P) from an activated uridine diphosphate (UDP)sugar to a molecule of the lipid carrier undecaprenylphosphate (Und-P) in the IM to form an Und-PP-sugar. This reaction is catalyzed by priming or initiating enzymes, which can be broadly classified into two protein families: the polyisoprenyl-phosphate hexose-1-phosphate transferases (PHPTs) or the polyisoprenyl-phosphate $N$-acetylhexosamine-1-phosphate transferases (PNPTs) (Lukose et al. 2017; Valvano 2011). Subsequently, the two pathways diverge. In the Wzx/Wzy-dependent pathway, glycosyltransferases (GTs) add sugars to the Und-PP-sugar molecule to synthesize the repeat unit. Each lipid-linked individual unit is translocated across the IM by the Wzx flippase and polymerized into the longer polysaccharide chain in the periplasm by the Wzy polymerase with the help of a polysaccharide co-polymerase (PCP) with chain elongation taking place at the reducing terminus of the Und-PP-linked polymer. Two families of PCPs (i.e., PCP-1 and PCP-2) are involved in regulating synthesis and chain length. While both are integral membrane proteins, the PCP-2 proteins contain an additional tyrosine kinase domain either located in the $\mathrm{C}$-terminus of the protein or as a separate protein (Whitfield et al. 2020).
By contrast, in the $\mathrm{ABC}$ transporter-dependent pathway, GTs act sequentially to transfer sugar molecules to the distal end of the growing Und-PP-linked polysaccharide chain to generate the complete polysaccharide chain of repeat units in the cytoplasm. Subsequently, the completed chain is translocated across the IM to the periplasm by an $\mathrm{ABC}$ transporter (Greenfield and Whitfield 2012; Whitfield et al. 2020). In the ABC transporter-dependent pathways, chain extension can be regulated by proteins of the WbdD methyltransferase/ kinase-methyltransferase family that add a terminal modification (i.e. methyl or methyl-phosphate) in systems where the ATPase of the involved ABC transporter contains a C-terminal carbohydrate binding domain (e.g. Escherichia coli serotypes 08, 09 and 09a) (Greenfield and Whitfield 2012). Alternatively, in systems lacking this terminal modification, it has been suggested that length could be controlled through the coupling of synthesis and transport (Greenfield and Whitfield 2012).

In both Wzx/Wzy- and $\mathrm{ABC}$ transporter-dependent pathways, the polysaccharide chain reaches the cell surface by translocation through an OM polysaccharide export (OPX) protein (Cuthbertson et al. 2009). In the specific case of O-antigen synthesized by such a pathway, it is transported as described below after ligation to lipid A-core.

In the synthase dependent pathway, polymerization and transport to the periplasm occur simultaneously by a synthase protein and transport to the cell-surface depends on a $\beta$-barrel protein in the $\mathrm{OM}$ together with a periplasmic TPR-rich protein which can be part of the $\beta$-barrel protein or a separate protein (Whitfield et al. 2020).

\section{Multicellular lifecycle of $\boldsymbol{M}$. xanthus}

M.xanthus is a predatory rod-shaped bacterium with a social lifecycle that is regulated by nutrient availability (Figure 2A). In the presence of nutrients, cells grow, divide and move across surfaces to form coordinately-spreading colonies (Schumacher and Sogaard-Andersen 2017; Zhang et al. 2012). In response to nutrient starvation, $M$. xanthus initiates a multicellular developmental program that culminates in the formation of spore-filled fruiting bodies (Konovalova et al. 2010). In this program, cells initially aggregate to form small aggregation centers and, as cells continue to accumulate, these centers increase in size and eventually become mound-shaped. By $24 \mathrm{~h}$, the aggregation process is complete and cells that have accumulated inside the nascent fruiting bodies differentiate into spherical spores resulting in mature fruiting bodies. Myxospores germinate in the presence of nutrients, thus, closing the cycle. M. xanthus is also a 


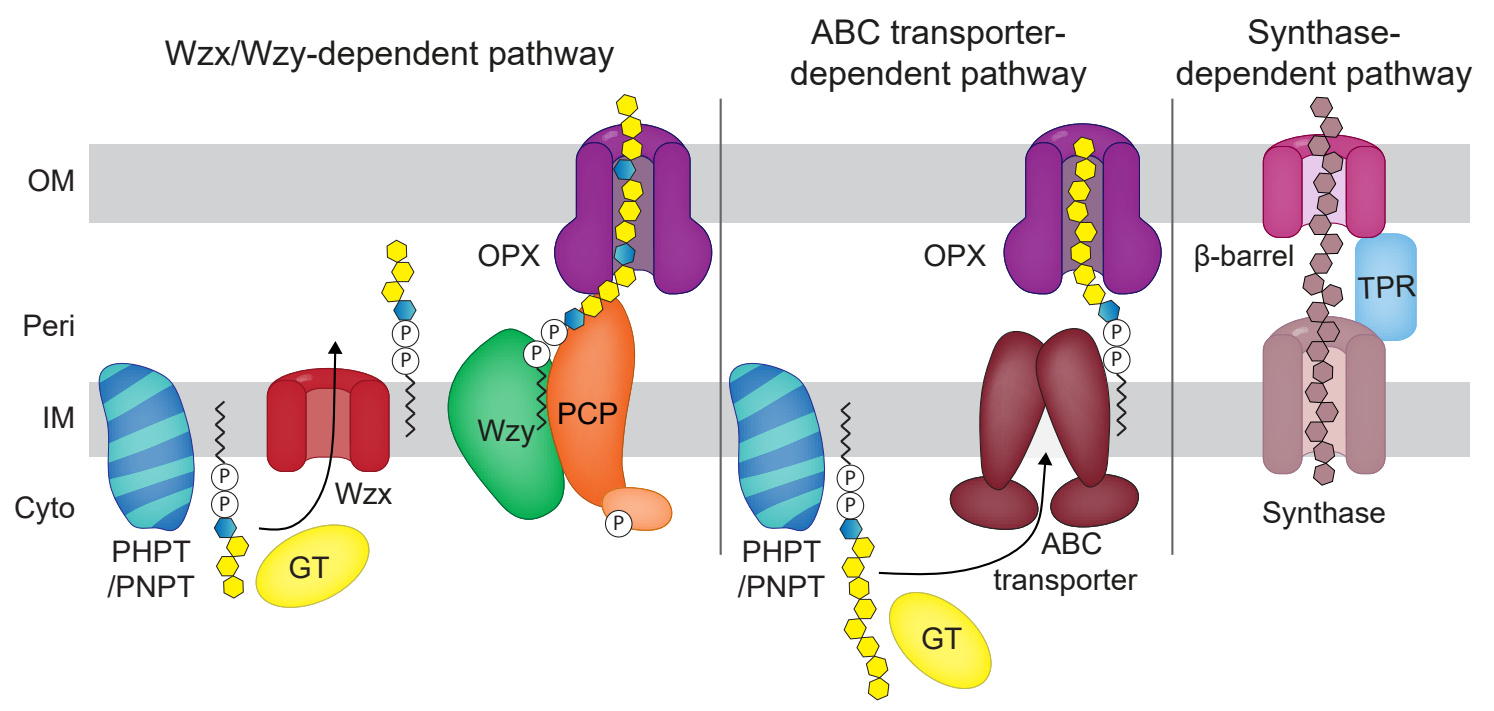

Figure 1: General overview of the Wzx/Wzy-, the $A B C$ transporter- and the synthase-dependent pathways for surface polysaccharides biosynthesis in Gram-negative bacteria.

For simplicity only one GT is shown per pathway. In the schematic of the Wzx/Wzy-pathway, the light orange symbol indicates the tyrosine kinase associated with some PCPs. See text for details.

predator that can prey on other microorganisms in what has been described as a wolf pack-like manner whereby M.xanthus cells jointly penetrate prey colonies and lyse prey cells (Berleman and Kirby 2009). The execution of these social activities depends on cell motility and its regulation as well as on cell-surface polysaccharides. $M$. xanthus cells have two motility systems for translocation across surfaces (Hodgkin and Kaiser 1979). One depends on type IV pili (T4P), which undergo cycles of extension, adhesion and retraction to pull cells forward (Craig et al. 2019), and one is for gliding motility and depends on the Agl-Glt complexes (Schumacher and Sogaard-Andersen 2017; Zhang et al. 2012). Both systems are polarized and only assemble at the leading pole of the rod-shaped cells (Figure 2B). Motility is regulated by controlling the cellular reversal frequency (Blackhart and Zusman 1985). During reversals, which are induced by the Frz chemosensory system (Mercier and Mignot 2016), cells stop and then resume movement in the opposite direction with the old leading pole becoming the new lagging pole (Figure 2B).

The four cell-surface polysaccharides synthesized by $M$. xanthus include (1) a polysaccharide referred to as exopolysaccharide (EPS), (2) a recently discovered polysaccharide with biosurfactant properties and referred to as biosurfactant polysaccharide (BPS), (3) a spore coat polysaccharide, and (4) the O-antigen of LPS. M. xanthus cells translocating across a surface generate a slime trail, which by lectin staining was suggested to contain sugar molecules (Ducret et al. 2012, 2013). However, the nature and synthesis of slime remain unknown (see below).
EPS, BPS and the spore coat polysaccharide are synthesized by dedicated Wzx/Wzy-dependent pathways and the O-antigen is synthesized by an ABC transporterdependent pathway. EPS, BPS, and O-antigen-capped LPS are important for motility and development (Bowden and Kaplan 1998; Islam et al. 2020; Lu et al. 2005; PérezBurgos et al., 2019, 2020a), while the spore coat polysaccharide is specifically required for formation of the environmentally-resistant spores (Holkenbrink et al. 2014; Müller et al. 2012; Pérez-Burgos et al. 2020b).

\section{Biosynthesis and function of EPS}

M. xanthus cells are surrounded by an extracellular matrix composed of EPS and proteins in a ratio of approximately 1:1 (Behmlander and Dworkin 1994). Initially, it was proposed that EPS was a capsular polysaccharide (Arnold and Shimkets 1988a; Behmlander and Dworkin 1994; Kim et al. 1999; Merroun et al. 2003); however, recent evidence supports a model whereby it is only loosely associated with the cell surface (Berleman et al. 2016; Gloag et al. 2016; Hu et al. 2013). The monosaccharide composition of EPS was reported in three studies with different results, but the common components include glucose, galactose and glucosamine (Behmlander and Dworkin 1994; Islam et al. 2020; Sutherland and Thomson 1975). The structure of EPS remains unknown.

Biosynthesis of EPS occurs via a Wzx/Wzy-dependent pathway and the core components of this pathway are 


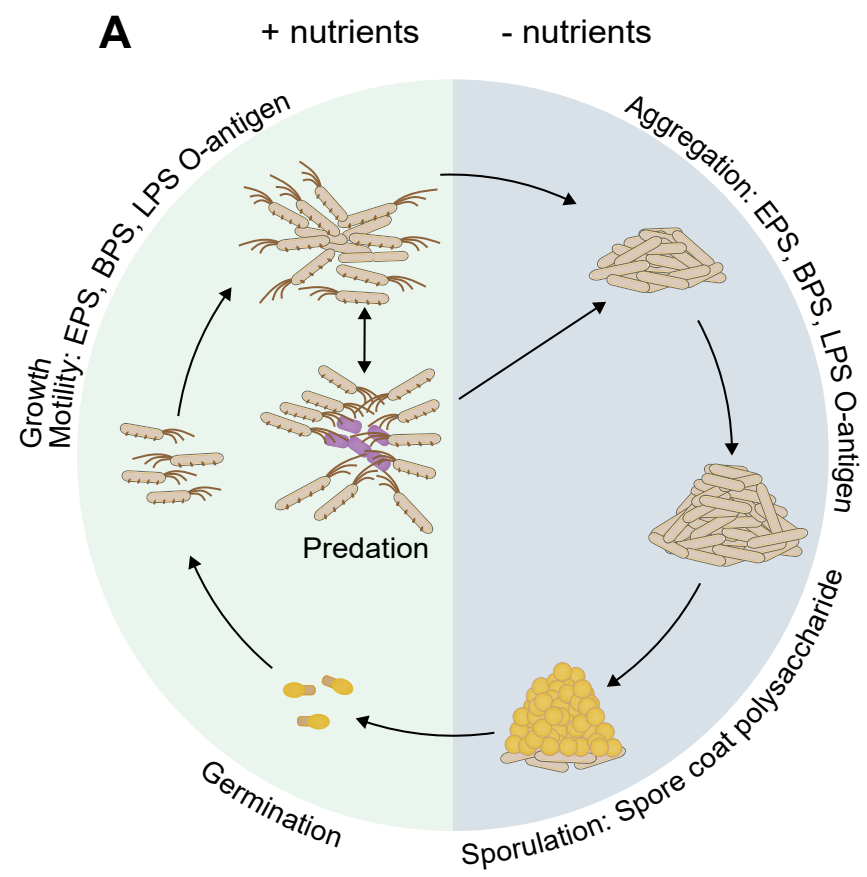

B
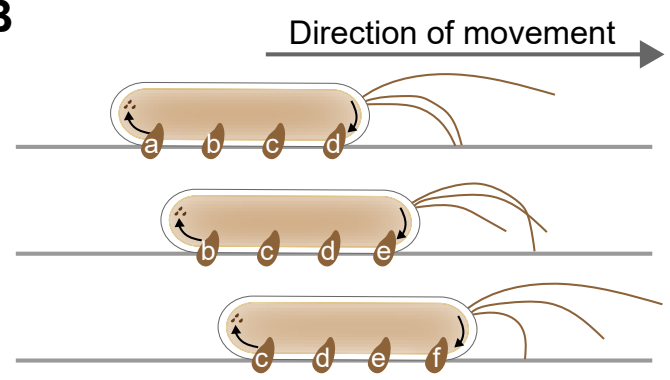

Frz system
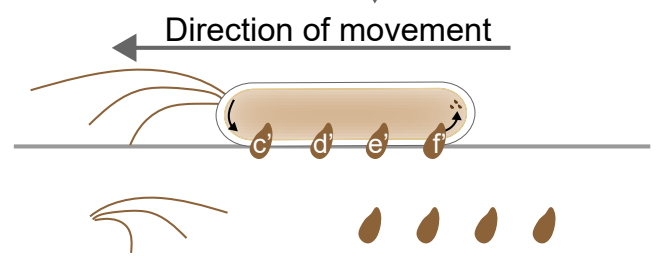

Type IV pili

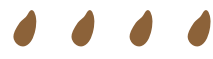

Agl-Glt complexes for gliding motility
Figure 2: M. xanthus life cycle and motility. (A) The life cycle of $M$. xanthus is regulated by nutrient availability. In the predation diagram, purple cells indicate prey cells. (B) M. xanthus has two polarized motility systems. T4P are extended at the leading cell pole and the Agl-Glt complexes for gliding motility are assembled at the leading cell pole (bent arrow), adhere to the substratum, and disassemble at the lagging cell pole (upwards bent arrow) (Schumacher and SogaardAndersen 2017; Zhang et al. 2012). In response to signaling by the Frz chemosensory system, cells reverse their direction of movement and the motility machineries become active at the new leading cell pole (Mercier and Mignot 2016). encoded by the eps locus (Berleman et al. 2011; Islam et al. 2020; Lu et al. 2005; Pérez-Burgos et al. 2020a; Zhou and Nan 2017). In addition, the eps locus encodes five GTs and a serine O-acetyltransferase (Figure 3A) (Islam et al. 2020; Pérez-Burgos et al. 2020a), several of which were shown to be important for EPS biosynthesis (Lu et al. 2005). Based on heterologous expression experiments in Salmonella enterica, the EpsZ protein is a PHPT with Galactose-1-P (Gal-1-P) transferase activity and initiates synthesis of the EPS repeat unit likely by transferring Gal-1-P to Und-P. In the current model, the repeat unit is then extended by the action of the five GTs (EpsU, EpsH, EpsE, EpsD, EpsA) and, subsequently, individual repeat units are translocated across the IM to the periplasm by the $\mathrm{Wzx}_{\mathrm{EPS}}$ flippase. Next, the Wzy $y_{\text {EPS }}$ polymerase with the help of the PCP protein EpsV polymerizes the repeat units to generate the high molecular weight polysaccharide, which is transported to the surface via the OPX protein (EpsY). It has been also suggested that 


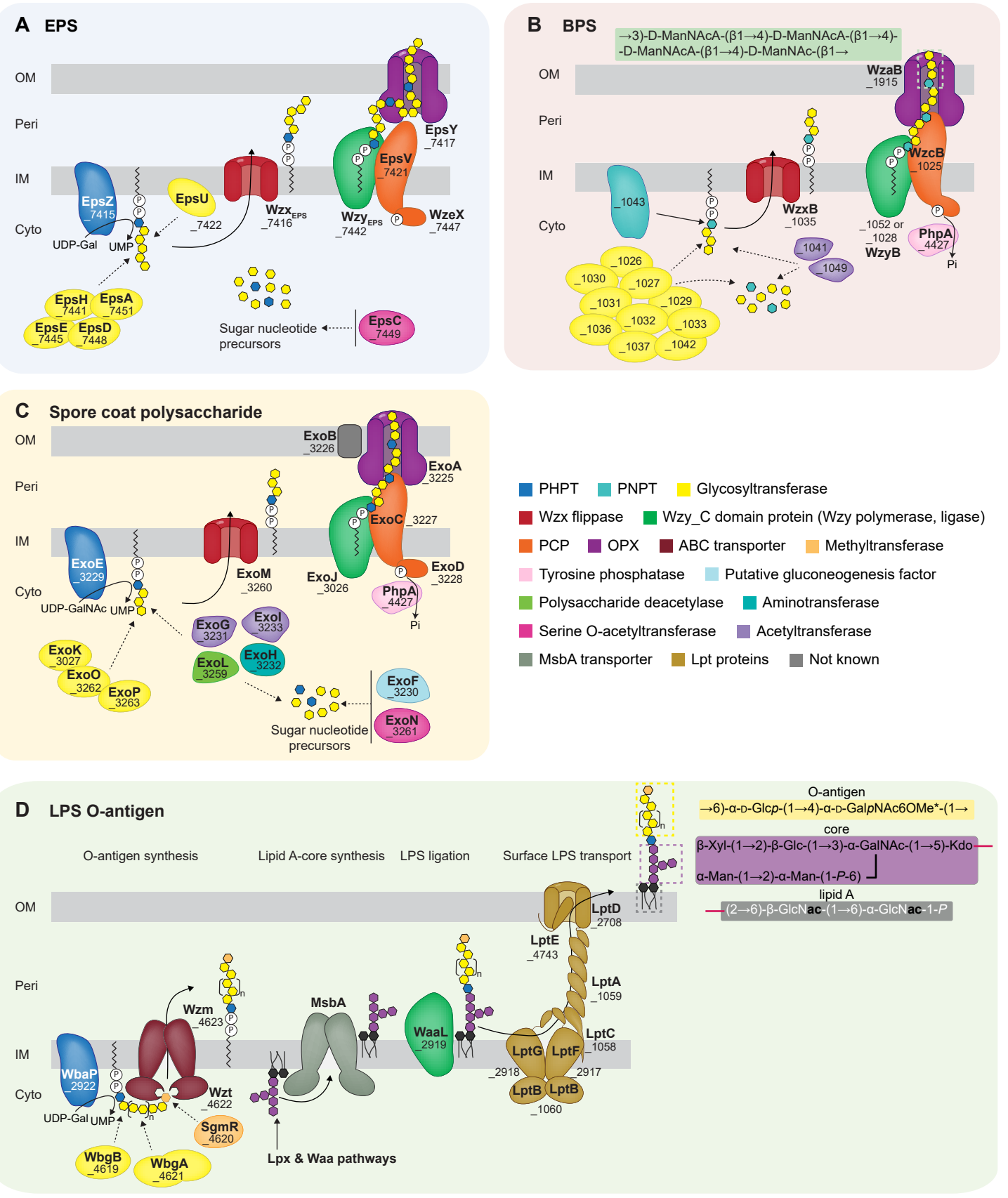

Figure 3: Biosynthesis of EPS, BPS, spore coat polysaccharide, and LPS 0-antigen in M. xanthus.

See text for details. In (A), tyrosine kinase activity of WzeX has been suggested but not shown. Structure of $M$. xanthus BPS (B) and LPS (D) is indicated. In (B), ManNACA is short for $\mathrm{N}$-acetyl-D-mannosaminuronic acid. In (D), ac in bold/black in the lipid A structure is short for 13-methylC14-30H (iso-C15-30H), C16-30, or 15-methyl-C16-30H (iso-C17-30H) based on Maclean et al. (2007), Man and Xyl are short for mannose and xylose, respectively. In (D) the red line indicates how core is linked to lipid A. Color code indicates function of proteins._XXXX is short for MXAN_XXXX protein identifiers.

WzeX is a stand-alone tyrosine kinase that forms part of a bipartite PCP together with EpsV. Additionally, the serine O-acetyltransferase EpsC is thought to be involved in sugar nucleotide precursor biosynthesis (Islam et al. 2020; PérezBurgos et al. 2020a).
Generally, there is agreement that EPS is important for cell-cell cohesion, T4P-dependent motility, and development. In suspension, $M$. xanthus cells cohere to form macroscopic aggregates that agglutinate and mutants lacking EPS $\left(\mathrm{EPS}^{-}\right)$are defective in cohesion and 
aggregation (Arnold and Shimkets 1988a, b; Hu et al. 2012b; Pérez-Burgos et al. 2020a; Shimkets 1986b; Yang et al. 2000b). Mutants with an $\mathrm{EPS}^{-}$phenotype have reduced T4P-dependent motility (Berleman et al. 2016; Islam et al. 2020; Li et al. 2003; Lu et al. 2005; Pérez-Burgos et al. 2020a; Yang et al. 2000a; Zhou and Nan 2017); however, detailed analyses suggest that the underlying mechanisms causing this motility defect are different in different mutants. Three different mechanisms have been proposed for the connection between EPS and/or the EPS biosynthetic pathway and stimulation of T4P-dependent motility:

(i) First, mutants lacking any one of the five core components of the Wzx/Wzy pathway for EPS biosynthesis ( $\Delta$ eps mutants), are hypo-piliated, and the few pili formed are able to retract (Pérez-Burgos et al. 2020a). These observations suggest that EPS and/or the EPS biosynthetic protein(s) are important for T4P extension (Pérez-Burgos et al. 2020a).

(ii) Second, EPS ${ }^{-}$strains with mutations in genes for the Dif chemosensory system, which is an important regulator of EPS biosynthesis (see below) (Bellenger et al. 2002; Yang et al. 1998a), make T4P at WT levels or are hyper-piliated (Li et al. 2003; Yang et al. 2000b). Importantly, addition of isolated EPS to a $\triangle$ difA mutant, which has an $\mathrm{EPS}^{-}$phenotype, caused reduced piliation (Hu et al. 2011; Li et al. 2003). It has also been reported that T4P as well as the major pilin PilA bind to extracted EPS ( $\mathrm{Hu}$ et al. 2012a; Li et al. 2003). Because dif mutants with an $\mathrm{EPS}^{-}$phenotype make T4P but have reduced T4P-dependent motility, these observations altogether suggest that EPS per se (rather than the EPS biosynthetic protein(s)) might promote T4P-dependent motility by stimulating T4P retraction (Hu et al. 2012b; Li et al. 2003).

(iii) Third, it has been proposed that EPS is involved in regulating the cell reversal frequency: A $\Delta e p s Z$ mutant was reported to have an increased reversal frequency (Zhou and Nan 2017) while dif mutants with an EPS phenotype were reported to have decreased reversal frequencies (Kearns et al. 2000; Shi et al. 2000).

The three mechanisms for the connection between EPS and/or the EPS biosynthetic pathway and T4P-dependent motility were obtained using different mutations and different WT strains, and mutants were tested under different conditions. Nevertheless, they support the idea that EPS and/or the EPS biosynthetic pathway may interface with the T4P machinery via several mechanisms.

Concerning fruiting body formation and sporulation, and as for T4P-dependent motility, different results have been obtained for different $\mathrm{EPS}^{-}$mutants. The biosynthetic
$\Delta$ eps mutants have a conditional defect in development: They develop and form fruiting bodies on a solid surface with only a slight delay and sporulate as WT (Berleman et al. 2016; Islam et al. 2020; Pérez-Burgos et al. 2020a). However, under submerged conditions, the $\Delta$ eps mutants have an aggregation defect likely due to the lack of cellcell cohesion and agglutination and, therefore, these mutants also have a sporulation defect under these conditions (Pérez-Burgos et al. 2020a). By contrast, other mutants with an EPS $^{-}$phenotype, generally, do not aggregate to make fruiting bodies and do not sporulate under any condition tested (Bellenger et al. 2002; Downard et al. 1993; Lancero et al. 2002; Pham et al. 2005; Ramaswamy et al. 1997; Shimkets 1986a; Yang et al., 1998b, 2000a). Moreover, addition of extracted EPS to EPS ${ }^{-}$dif mutants, rescued these developmental defects (Chang and Dworkin 1994; Yang et al. 2000b). As for the experiments in which isolated EPS was found to stimulate T4P retraction in $\mathrm{EPS}^{-}$dif mutants, these experiments suggest that EPS is directly involved in development.

In contrast to the recently described biosynthetic pathway for EPS (Islam et al. 2020; Pérez-Burgos et al. 2020a), many mutants with mutations in regulatory genes and with altered EPS biosynthesis have been described (Figure 4). At the molecular level, it is not known how these regulators function to affect EPS synthesis. Therefore, it remains an open question whether the effect of these mutations is directly on EPS synthesis or indirect by affecting other processes. Among the different regulators, the Dif chemosensory system (Yang et al. 1998a; Yang and Li 2005) is the best-characterized. DifA (a methyl-accepting chemotaxis protein [MCP]), DifE (a CheA-type histidine kinase), DifC (a CheW coupling protein) together with the single-domain response regulator EpsW, which is phosphorylated by DifE, are positive regulators of EPS synthesis (Bellenger et al. 2002; Black et al. 2015; Lancero et al. 2005; Yang et al. 1998b; Yang and Li 2005) (Figure 4). The singledomain response regulator DifD is also phosphorylated by DifE and has been suggested to act as a phosphate sink for the phosphate flow from DifE and inhibits EPS synthesis (Black and Yang, 2004, 2010). DifD is dephosphorylated by the CheC-like phosphatase DifG, which is also an inhibitor of EPS synthesis (Black and Yang, 2004, 2010). DifE has also been suggested to interact with the NtrC-like transcriptional regulator Nla19 (Lancero et al. 2005); however, it is not known whether DifE phosphorylates Nla19. Genetically, Nla19 is an inhibitor of EPS synthesis (Lancero et al. 2005). How the Dif system regulates EPS synthesis is not known. The activity of the Dif system has been suggested to be regulated by the T4P machinery involved in extending and retracting T4P (Black et al., 2006, 2017; Wu 
and Kaiser 1995). Specifically, mutants of this machinery that are unable to make T4P have an $\mathrm{EPS}^{-}$phenotype while a $\triangle$ pilT mutant, which lacks the PilT retraction ATPase and is hyper-piliated, produces increased levels of EPS (Black et al., 2006, 2017; Bretl et al. 2016; Dana and Shimkets 1993; Shimkets 1986b). In the current model, the PilB ATPase for T4P extension is an important link between the T4P machinery and EPS synthesis (Black et al. 2017). The DnaK homolog StkA is a negative regulator of EPS synthesis and has also been implicated in linking the T4P machinery and EPS synthesis (Black et al. 2006; Dana and Shimkets 1993; Moak et al. 2015) (Figure 4). Thus, EPS and/or the EPS biosynthetic machinery is important for T4P formation and function and, inversely, the T4P machinery is important for EPS biosynthesis (Figure 4).

The second messenger c-di-GMP is a key regulator of the biosynthesis of secreted polysaccharides in many species (Römling et al. 2013). Recently, c-di-GMP has also emerged as involved in regulation of EPS biosynthesis in $M$. xanthus (Figure 4). C-di-GMP accumulates at the same level in exponentially growing and stationary phase $M$. xanthus cells while the level increases approximately 10-fold during development (Skotnicka et al., 2015, 2016). Lack of the diguanylate cyclase DmxA causes an increase in EPS biosynthesis and in the cellular level of c-di-GMP during growth (Skotnicka et al. 2015). Moreover, lack of SgmT, which contains an enzymatically inactive diguanylate cyclase domain (GGDEF domain) but is still able to bind c-di-GMP (Petters et al. 2012; Skotnicka et al. 2015), or its cognate response regulator DigR causes an increase in EPS synthesis as well as in the cellular level of c-di-GMP during growth (Overgaard et al. 2006; Petters et al. 2012; Skotnicka et al. 2015). Also, lack of the histidine kinase TmoK, which has an enzymatically inactive GGDEF domain and does not bind c-di-GMP, caused an increase in EPS biosynthesis without altering the c-di-GMP level during growth (Skotnicka et al. 2015). How these proteins and c-di-GMP interface with EPS synthesis is not known. The diguanylate cyclase $\mathrm{DmxB}$ is specifically synthesized during development and is responsible for the increase in c-diGMP during development (Skotnicka et al. 2016). DmxB is also important for the increased EPS synthesis during development. During development, c-di-GMP has been suggested to bind to the NtrC-like transcriptional regulator Nla24 to stimulate transcription of eps genes (Skotnicka et al. 2016).

\section{Biosynthesis and function of BPS}

BPS is a recently discovered secreted polysaccharide in M. xanthus (Islam et al. 2020) that consists of randomly acetylated tetrasaccharide repeats composed of $\mathrm{N}$-acetylmannosaminuronic acid (ManNAcA) and $\mathrm{N}$ -

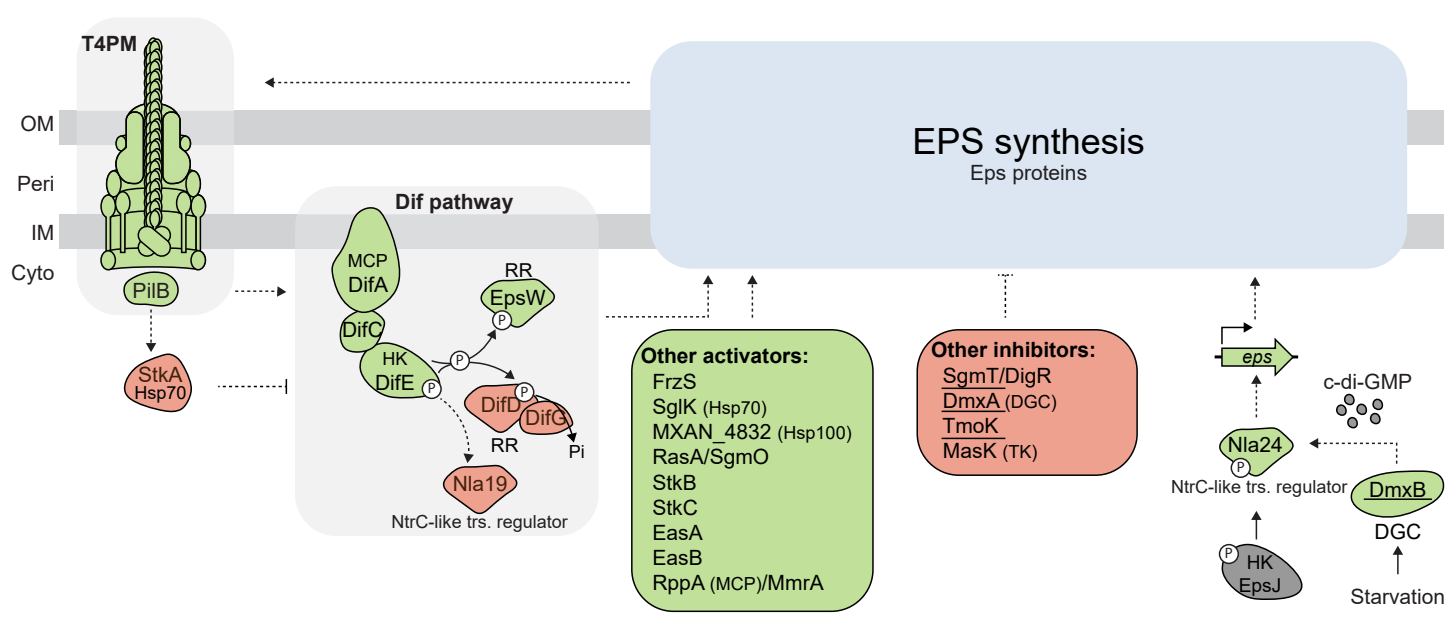

Figure 4: Regulation of EPS synthesis.

Green and red, activators and inhibitors of EPS synthesis, respectively; gray, the histidine kinase Eps) phosphorylates Nla24 in vitro (Willett et al. 2013) but its effect on EPS synthesis is not known. Stippled line, interaction mechanism unknown; continuous line, direct interaction. For the connection between T4P, the T4P machinery (T4PM), the Dif chemosensory system and the DnaK homolog StkA, see main text for details. Underlined proteins indicate proteins with a GGDEF domain among which only DmxA and DmxB have diguanylate cyclase (DGC) activity. For the connection between c-di-GMP and EPS synthesis, see main text for details. Abbreviations used: RR, single-domain response regulator, HK, histidine kinase, MCP (methyl-accepting chemotaxis protein), TK (tyrosine kinase). References for these regulators are: MasK (Thomasson et al. 2002), FrzS (Berleman et al. 2011), SglK (Weimer et al. 1998; Yang et al. 1998a), MXAN_4832 (Yan et al. 2012), RasA/SgmO (Pham et al. 2005; Youderian and Hartzell 2006), StkB and StkC (Moak et al. 2015), EasA and EasB (Lu et al. 2005), RppA and MmrA (Kimura et al. 2004). 
acetylmannosamine (ManNAc) (Figure 3B) (Islam et al. 2020). BPS is suggested to be released by cells (Islam et al. 2020). Biosynthesis of BPS involves a Wzx/Wzy-dependent pathway, which is encoded by two loci (Islam et al. 2020; Pérez-Burgos et al. 2020a) (Figure 3B). Repeat unit synthesis is initiated by the PNPT MXAN_1043, and then suggested to be extended by different glycosyltransferases. The repeat units are translocated to the periplasm via the flippase MXAN_1035 (WzxB) and polymerized through the action of the Wzy polymerase MXAN_1052 or, alternatively, MXAN_1028 (WzyB). Transport across the OM occurs through the OPX protein MXAN_1915 (WzaB) with the help of the PCP MXAN_1025 (WzcB, also referred to as BtkB (Kimura et al. 2012)) (Islam et al. 2020; Pérez-Burgos et al. 2020a). The tyrosine phosphatase PhpA is thought to dephosphorylate phosphorylated $\mathrm{WzcB} / \mathrm{BtkB}$ (Mori et al. 2012).

BPS has biosurfactant activity and was suggested to stimulate T4P-dependent motility by reducing surface tension (Figure 2A) (Islam et al. 2020). Moreover, bps mutants were reported to form fruiting bodies at a lower cell density than WT (Islam et al. 2020) but it is not known whether BPS is important for sporulation. T4P were suggested to inhibit BPS synthesis (Islam et al. 2020) while it remains unknown whether BPS and/or the BPS biosynthetic machinery is important for T4P formation. Interestingly, Islam et al. (2020) also reported that BPS is predominantly synthesized by cells at the center of a spreading colony while EPS is synthesized by cells towards the periphery.

\section{Biosynthesis of the spore coat polysaccharide}

During development, the rod-shaped M. xanthus cells that have accumulated inside fruiting bodies differentiate to environmentally resistant spherical spores (Figure 2A) (Dworkin and Voelz 1962). During spore differentiation, PG is thought to be removed (Bui et al. 2009) and replaced by a highly resistant spore coat that is mainly composed of polysaccharide (Holkenbrink et al. 2014; Kottel et al. 1975) but also contains several proteins (Inouye et al. 1979a; Leng et al. 2011; McCleary et al. 1991). The spore coat protects the cells from environmental stresses (e.g., heat, desiccation, ultraviolet light and sonication) (Sudo and Dworkin 1969). None of the proteins in the spore coat are essential for spore formation (Curtis et al. 2007; Inouye et al. 1979b; Komano et al. 1984; Lee et al. 2011; Leng et al. 2011), while the spore coat polysaccharide is.
The spore coat polysaccharide is composed of 1-3-, 14-linked $N$-acetylgalactosamine (GalNAc), 1-4-linked glucose (Glc) and additionally contains glycine, which was suggested to form glycine bridges between the polysaccharide chains (Holkenbrink et al. 2014). Synthesis and export of the spore coat polysaccharide depend on the ExoA-P proteins, which constitute a Wzx/Wzy pathway exclusively dedicated to this process (Holkenbrink et al. 2014; Islam et al. 2020; Pérez-Burgos et al. 2020b) (Figure 3C). The NfsA-H proteins, powered by the AglQRS proteins in a proton motive force-dependent manner, are also important for sporulation and modify the Exo generated polysaccharide to generate the rigid, stress-bearing spore coat (Holkenbrink et al. 2014; Müller et al. 2012; Wartel et al. 2013). Based on chemical analysis of the spore coat polysaccharide in the absence and presence of the Nfs proteins, this modification involves an alteration in the chain length of the spore coat polysaccharide (Holkenbrink et al. 2014).

The ExoA-P proteins are encoded by three loci (Holkenbrink et al. 2014; Islam et al. 2020; Pérez-Burgos et al. $2020 b)$. In the current model, ExoE is the priming enzyme for repeat unit biosynthesis (Holkenbrink et al. 2014; PérezBurgos et al. 2020b) (Figure 3C). Based on heterologous expression experiments in E. coli, ExoE likely transfers GalNAc-1-P to Und-P despite belonging to the PHPT family of priming enzymes (Pérez-Burgos et al. 2020b). Three GTs (ExoK, ExoO and ExoP) synthesize the repeat unit, which is likely a tetrasaccharide composed of GalNAc and Glc (Holkenbrink et al. 2014; Pérez-Burgos et al. 2020b) (Figure 3C). Additionally, the $\mathrm{N}$-acetyltransferase homologs ExoG and ExoI, the aminotransferase homolog ExoH, and the polysaccharide deacetylase homolog ExoL could modify sugars before or after incorporation into the repeat unit (Figure 3C). For instance, based on their homology to the FemX protein that is involved in addition of glycine to the lipid II PG precursor in Staphylococcus aureus (Favrot et al. 2016), ExoG and ExoI were suggested to be involved in glycine addition to the repeat unit by transferring glycine to the amino group added by ExoH (Pérez-Burgos et al. 2020b). Moreover, ExoF (Holkenbrink et al. 2014) and ExoN (Pérez-Burgos et al. 2020b) have been suggested to be involved in synthesis of precursors important for synthesis of the repeat unit (Figure 3C). Translocation of the repeat unit to the periplasm occurs via the ExoM flippase, followed by polymerization of the repeat units by the Wzy polymerase, ExoJ (Islam et al. 2020; Pérez-Burgos et al. 2020b). Polymerization is thought to be controlled by the bipartite PCP formed by the transmembrane protein ExoC and the cytoplasmic ExoD tyrosine kinase (Holkenbrink et al. 2014; Kimura et al. 2011).The tyrosine phosphatase 
PhpA dephosphorylates the phosphorylated ExoD/BtkA protein (Mori et al. 2012). The polysaccharide polymer is transported to the cell-surface via the OPX protein homolog ExoA. The OM protein ExoB is important for spore coat polysaccharide synthesis (Holkenbrink et al. 2014), but its precise function in this process is not known.

An intriguing aspect of the spore differentiation process is that the PG is likely removed (Bui et al. 2009) and replaced with the spore coat (Müller et al. 2012). It has been suggested that the spore coat protects cells from bursting due to intracellular turgor in the absence of PG (Bui et al. 2009). Interestingly exo and $n f s$ mutants, which cannot synthesize the intact spore coat, initiate the spore differentiation process and become shorter and wider (Holkenbrink et al. 2014; Müller et al. 2012; Pérez-Burgos et al. 2020b). However, at some point the spore differentiation process is aborted and cells either return to rod-shape or remain morphologically highly abnormal (Holkenbrink et al. 2014; Müller et al. 2012; Pérez-Burgos et al. 2020b) suggesting that $\mathrm{PG}$ removal and spore coat polysaccharide synthesis are closely coupled.

\section{Biosynthesis and function of LPS 0-antigen}

Biosynthesis and transport of O-antigen and lipid A-core occur in separate pathways, and the two moieties are joined on the periplasmic side of the IM and then transported to the OM via the Lpt (LPS transport) system (Sperandeo et al. 2019; Whitfield and Trent 2014) (Figure 3D). The structures of the M. xanthus LPS O-antigen and lipid A-core have been elucidated (Maclean et al. 2007) (Figure 3D).

In $M$. xanthus, synthesis of O-antigen depends on an ABC transporter-dependent pathway (Guo et al. 1996) (Figure 3D), which is encoded by two loci and dedicated to this process (Pérez-Burgos et al. 2019). O-antigen synthesis is primed by the PHPT homolog WbaP, which based on heterologous expression experiments in S. enterica, transfers Gal-1-P to Und-P (Pérez-Burgos et al. 2019). The GTs WbgA and WbgB are involved in O-antigen synthesis (Guo et al. 1996; Yang et al. 2000a). It has been suggested that WbgB adds $a$-D-Glcp to the priming Gal residue of UndPP-Gal, while WbgA extends the polymer by alternatively transferring $a$-Gal $p$ NAc and $a$-D-Glc $p$ in successive cycles of catalysis (Pérez-Burgos et al. 2019). To terminate chain extension, the methyltransferase homolog SgmR has been suggested to methylate $a$-GalpNAc (Pérez-Burgos et al. 2019), consistent with the structure and composition of the
O-antigen (Maclean et al. 2007). Subsequently, the ABC transporter composed of Wzm and Wzt translocates the full-length Und-PP-linked O-antigen chain across the IM to the periplasm (Guo et al. 1996; Pérez-Burgos et al. 2019). Similarly to the E. coli $\mathrm{O}_{8} / \mathrm{O}_{9}$ systems (Greenfield and Whitfield 2012), the C-terminal carbohydrate binding domain of Wzt may recognize the modified terminus prior to transport of the O-antigen chain to the periplasm. The WaaL ligase (Pérez-Burgos et al. 2019) forms a glycosidic bond between the core part of the lipid A-core and the first sugar in the O-antigen to form the mature LPS molecule (Figure 3D). Finally, the LPS molecules are transported from the periplasmic side of the IM to the cell-surface via the Lpt pathway (Figure 3D). Generally, lipid A-core biosynthesis depends on the Lpx and Waa pathways (Raetz pathway) (Raetz and Whitfield 2002; Whitfield and Trent 2014) and the lipid A-core is translocated across the IM by the MsbA ABC transporter to the periplasm. Based on bioinformatic analyses, the Raetz and Lpt pathways are conserved in M. xanthus (Pérez-Burgos et al. 2019); however, they have not been analyzed experimentally.

The function of LPS O-antigen in M. xanthus has been controversial. However, using a $\triangle w b a P$ mutant, which is specifically blocked in the initiation of O-antigen synthesis, it was recently shown that LPS O-antigen is essential for gliding motility and fruiting body formation (Pérez-Burgos et al. 2019). In addition, cells of the $\triangle w b a P$ mutant had reduced T4P-dependent motility while assembling T4P at slightly higher levels than in WT (Pérez-Burgos et al. 2019). How LPS O-antigen affects these processes remains unknown.

\section{The enigmatic $M$. xanthus slime}

When translocating across a surface, $M$. xanthus cells leave behind a phase-bright trail that can be visualized with phase-contrast microscopy (Gloag et al. 2016; Wolgemuth et al. 2002). These trails are typically referred to as slime trails. Other M. xanthus cells tend to follow these trails independently of which motility system is active (Ducret et al. 2012; Gloag et al. 2016; Wolgemuth et al. 2002). Accordingly, it has been suggested that this trail-following behavior helps $M$. xanthus cells to coordinate their multicellular behaviors (Ducret et al. 2012; Gloag et al. 2016). It has also been suggested that slime may function as an adhesive for the Agl-Glt complexes for gliding motility (Ducret et al. 2012). For many years, major outstanding questions have been the nature, chemical composition and synthesis of slime. Recently, some progress has been made. First, slime can be stained using the lectin Concanavalin A 
(Ducret et al. 2012), the lipophilic dye FM1-43FX (Gloag et al. 2016), and acridine orange (Wolgemuth et al. 2002) suggesting that slime contains sugar(s), lipid(s) and extracellular DNA (eDNA). Moreover, outer membrane vesicles, which are produced in large amounts by $M$. xanthus (Kahnt et al. 2010), were also identified in slime trails using transmission electron microscopy (Ducret et al. 2013). Interestingly, Gloag et al. (2016) also reported that translocating $M$. xanthus cells generate phase-bright furrows in the underlying substratum. Altogether, these observations suggest that the phase bright trails are the result of physical deformation of the underlying substratum and the deposition of different chemical components, i.e., slime, of $M$. xanthus cells.

It has been suggested that slime is likely deposited from beneath cells (Ducret et al. 2012). However, it is not known whether this is a passive process and/or whether these components are actively secreted. Concerning the saccharidic content of slime, it has been detected using Concanavalin A staining in a wzm wzt wbgA triple mutant, which lacks $\mathrm{O}$-antigen, in a difA mutant, which is deficient in EPS synthesis, and in a $\triangle w z a B$ mutant, which lacks BPS, as well as in a triple mutant that lacks all three OPX proteins of the three Wzx/Wzy pathways in M. xanthus (Ducret et al. 2012) (Figure 3A-C). These experimental data indicate that none of the four identified biosynthetic machineries is single-handedly responsible for producing the saccharidic component of slime. However, it remains a possibility that the saccharidic component is a mixture of BPS, EPS and LPS. Alternatively, a yet to be identified pathway could be involved in producing the saccharidic component of slime.

\section{Concluding remarks}

Recently, major progress has been made in our understanding of the biosynthetic pathways for EPS, BPS, spore coat polysaccharide and the O-antigen of LPS in M. xanthus. This work not only provides an important foundation to understand in detail the function of EPS, BPS and O-antigen capped LPS in the social life cycle of $M$. xanthus but also form the basis to elucidate the precise composition and structure of these polysaccharides. Also, it will be of interest to disentangle the regulatory relationship between the T4P machinery and EPS biosynthesis to understand how EPS and/or the EPS biosynthetic machinery stimulates T4P formation and function as well as how the T4P machinery interfaces with EPS synthesis. Also, a completely open question is how the spatial regulation of BPS and EPS synthesis is accomplished. It will also be of interest to address the mechanism coordinating PG removal and spore coat polysaccharide biosynthesis during sporulation. Finally, purification and characterization of the saccharidic component of slime would be highly interesting as a way forward to understand its origin and biosynthesis.

Acknowledgments: We are profoundly grateful to Miguel Valvano and Inmaculada García-Romero (WellcomeWolfson Institute for Experimental Medicine at Queen's University Belfast) for many helpful and inspiring discussions. This work was supported by Deutsche Forschungsgemeinschaft (DFG, German Research Council) within the framework of the SFB987 "Microbial Diversity in Environmental Signal Response" as well as by the Max Planck Society.

Author contribution: All the authors have accepted responsibility for the entire content of this submitted manuscript and approved submission.

Research funding: This work was supported by Deutsche Forschungsgemeinschaft (DFG, German Research Council) within the framework of the SFB987 "Microbial Diversity in Environmental Signal Response" as well as by the Max Planck Society.

Conflict of interest statement: The authors declare no conflicts of interest regarding this article.

\section{References}

Arnold, J.W. and Shimkets, L.J. (1988a). Cell surface properties correlated with cohesion in Myxococcus xanthus. J. Bacteriol. 170: 5771-5777.

Arnold, J.W. and Shimkets, L.J. (1988b). Inhibition of cell-cell interactions in Myxococcus xanthus by Congo red. J. Bacteriol. 170: 5765-5770.

Behmlander, R.M. and Dworkin, M. (1994). Biochemical and structural analyses of the extracellular matrix fibrils of Myxococcus xanthus. J. Bacteriol. 176: 6295-6303.

Bellenger, K., Ma, X., Shi, W., and Yang, Z. (2002). A CheW homologue is required for Myxococcus xanthus fruiting body development, social gliding motility, and fibril biogenesis. J. Bacteriol. 184: 5654-5660.

Berleman, J.E. and Kirby, J.R. (2009). Deciphering the hunting strategy of a bacterial wolfpack. FEMS Microbiol. Rev. 33: 942-957.

Berleman, J.E., Vicente, J.J., Davis, A.E., Jiang, S.Y., Seo, Y.E., and Zusman, D.R. (2011). FrzS regulates social motility in Myxococcus xanthus by controlling exopolysaccharide production. PLoS ONE 6: e23920.

Berleman, J.E., Zemla, M., Remis, J.P., Liu, H., Davis, A.E., Worth, A.N., West, Z., Zhang, A., Park, H., Bosneaga, E., et al. (2016). Exopolysaccharide microchannels direct bacterial motility and organize multicellular behavior. ISME J. 10: 2620-2632.

Black, W.P., Schubot, F.D., Li, Z., and Yang, Z. (2010). Phosphorylation and dephosphorylation among Dif chemosensory proteins 
essential for exopolysaccharide regulation in Myxococcus xanthus. J. Bacteriol. 192: 4267-4274.

Black, W.P., Wang, L., Davis, M.Y., and Yang, Z. (2015). The orphan response regulator EpsW is a substrate of the DifE kinase and it regulates exopolysaccharide in Myxococcus xanthus. Sci. Rep. 5: 17831.

Black, W.P., Wang, L.L., Jing, X., Saldana, R.C., Li, F., Scharf, B.E., Schubot, F.D., and Yang, Z.M. (2017). The type IV pilus assembly ATPase PilB functions as a signaling protein to regulate exopolysaccharide production in Myxococcus xanthus. Sci. Rep. 7: 7263. https://doi.org/10.1038/s41598-017-07594-x.

Black, W.P., Xu, Q., and Yang, Z. (2006). Type IV pili function upstream of the Dif chemotaxis pathway in Myxococcus xanthus EPS regulation. Mol. Microbiol. 61: 447-456.

Black, W.P. and Yang, Z.M. (2004). Myxococcus xanthus chemotaxis homologs DifD and DifG negatively regulate fibril polysaccharide production. J. Bacteriol. 186: 1001-1008.

Blackhart, B.D. and Zusman, D.R. (1985). "Frizzy" genes of Myxococcus xanthus are involved in control of frequency of reversal of gliding motility. Proc. Natl. Acad. Sci. USA 82: 8767-8770.

Bogdanov, M., Pyrshev, K., Yesylevskyy, S., Ryabichko, S., Boiko, V., Ivanchenko, P., Kiyamova, R., Guan, Z., Ramseyer, C., and Dowhan, W. (2020). Phospholipid distribution in the cytoplasmic membrane of Gram-negative bacteria is highly asymmetric, dynamic, and cell shape-dependent. Sci. Adv. 6: eaaz6333.

Bowden, M.G. and Kaplan, H.B. (1998). The Myxococcus xanthus lipopolysaccharide 0 -antigen is required for social motility and multicellular development. Mol. Microbiol. 30: 275-284.

Bretl, D.J., Müller, S., Ladd, K.M., Atkinson, S.N., and Kirby, J.R. (2016). Type IV-pili dependent motility is co-regulated by PilSR and PilS2R2 two-component systems via distinct pathways in Myxococcus xanthus. Mol. Microbiol. 102: 37-53.

Bui, N.K., Gray, J., Schwarz, H., Schumann, P., Blanot, D., and Vollmer, W. (2009). The peptidoglycan sacculus of Myxococcus xanthus has unusual structural features and is degraded during glycerolinduced myxospore development. J. Bacteriol. 191: 494-505.

Chang, B.Y. and Dworkin, M. (1994). Isolated fibrils rescue cohesion and development in the Dsp mutant of Myxococcus xanthus. J. Bacteriol. 176: 7190-7196.

Craig, L., Forest, K.T., and Maier, B. (2019). Type IV pili: dynamics, biophysics and functional consequences. Nat. Rev. Microbiol. 17: 429-440.

Curtis, P.D., Atwood, J., 3rd, Orlando, R., and Shimkets, L.J. (2007). Proteins associated with the Myxococcus xanthus extracellular matrix. J. Bacteriol. 189: 7634-7642.

Cuthbertson, L., Mainprize, I.L., Naismith, J.H., and Whitfield, C. (2009). Pivotal roles of the outer membrane polysaccharide export and polysaccharide copolymerase protein families in export of extracellular polysaccharides in Gram-negative bacteria. Microbiol. Mol. Biol. Rev. 73: 155-177.

Dana, J.R. and Shimkets, L.J. (1993). Regulation of cohesiondependent cell interactions in Myxococcus xanthus. J. Bacteriol. 175: 3636-3647.

Downard, J., Ramaswamy, S.V., and Kil, K.S. (1993). Identification of esg, a genetic locus involved in cell-cell signaling during Myxococcus xanthus development. J. Bacteriol. 175: 7762-7770.

Downie, J.A. (2010). The roles of extracellular proteins, polysaccharides and signals in the interactions of rhizobia with legume roots. FEMS Microbiol. Rev. 34: 150-170.
Ducret, A., Fleuchot, B., Bergam, P., and Mignot, T. (2013). Direct live imaging of cell-cell protein transfer by transient outer membrane fusion in Myxococcus xanthus. eLife 2: e00868.

Ducret, A., Valignat, M.P., Mouhamar, F., Mignot, T., and Theodoly, 0. (2012). Wet-surface-enhanced ellipsometric contrast microscopy identifies slime as a major adhesion factor during bacterial surface motility. Proc. Natl. Acad. Sci. USA 109: 10036-10041.

Dworkin, M. and Voelz, H. (1962). The formation and germination of microcysts in Myxococcus xanthus. J. Gen. Microbiol. 28: 81-85.

Favrot, L., Blanchard, J.S., and Vergnolle, O. (2016). Bacterial GCN5-related N-acetyltransferases: from resistance to regulation. Biochemistry 55: 989-1002.

Flemming, H.C., Wingender, J., Szewzyk, U., Steinberg, P., Rice, S.A., and Kjelleberg, S. (2016). Biofilms: an emergent form of bacterial life. Nat. Rev. Microbiol. 14: 563-575.

Gloag, E.S., Turnbull, L., Javed, M.A., Wang, H., Gee, M.L., Wade, S.A., and Whitchurch, C.B. (2016). Stigmergy co-ordinates multicellular collective behaviours during Myxococcus xanthus surface migration. Sci. Rep. 6: 26005.

Greenfield, L.K. and Whitfield, C. (2012). Synthesis of lipopolysaccharide $\mathrm{O}$-antigens by $\mathrm{ABC}$ transporter-dependent pathways. Carbohydr. Res. 356: 12-24.

Guo, D., Bowden, M.G., Pershad, R., and Kaplan, H.B. (1996). The Myxococcus xanthus rfbABC operon encodes an ATP-binding cassette transporter homolog required for 0 -antigen biosynthesis and multicellular development. J. Bacteriol. 178: 1631-1639.

Hodgkin, J. and Kaiser, D. (1979). Genetics of gliding motility in Myxococcus xanthus (Myxobacterales)-two gene systems control movement. Mol. Gen. Genet. 171: 177-191.

Holkenbrink, C., Hoiczyk, E., Kahnt, J., and Higgs, P.I. (2014). Synthesis and assembly of a novel glycan layer in Myxococcus xanthus spores. J. Biol. Chem. 289: 32364-32378.

Hu, W., Hossain, M., Lux, R., Wang, J., Yang, Z., Li, Y., and Shi, W. (2011). Exopolysaccharide-independent social motility of Myxococcus xanthus. PLoS ONE 6: e16102.

Hu, W., Lux, R., and Shi, W. (2013). Analysis of exopolysaccharides in Myxococcus xanthus using confocal laser scanning microscopy. Methods Mol. Biol. 966: 121-131.

Hu, W., Wang, J., McHardy, I., Lux, R., Yang, Z., Li, Y., and Shi, W. (2012a). Effects of exopolysaccharide production on liquid vegetative growth, stress survival, and stationary phase recovery in Myxococcus xanthus. J. Microbiol. 50: 241-248.

Hu, W., Yang, Z., Lux, R., Zhao, M., Wang, J., He, X., and Shi, W. (2012b). Direct visualization of the interaction between pilin and exopolysaccharides of Myxococcus xanthus with eGFP-fused PilA protein. FEMS Microbiol. Lett. 326: 23-30.

Inouye, M., Inouye, S., and Zusman, D.R. (1979a). Biosynthesis and self-assembly of protein $S$, a development specific protein of Myxococcus xanthus. Proc. Natl. Acad. Sci. USA 76: 209-213.

Inouye, M., Inouye, S., and Zusman, D.R. (1979b). Gene expression during development of Myxococcus xanthus: pattern of protein synthesis. Dev. Biol. 68: 579-591.

Islam, S.T., Vergara Alvarez, I., Saidi, F., Giuseppi, A., Vinogradov, E., Sharma, G., Espinosa, L., Morrone, C., Brasseur, G., Guillemot, J.F., et al. (2020). Modulation of bacterial multicellularity via spatio-specific polysaccharide secretion. PLoS Biol. 18: e3000728.

Kahnt, J., Aguiluz, K., Koch, J., Treuner-Lange, A., Konovalova, A., Huntley, S., Hoppert, M., Søgaard-Andersen, L., and Hedderich, 
R. (2010). Profiling the outer membrane proteome during growth and development of the social bacterium Myxococcus xanthus by selective biotinylation and analyses of outer membrane vesicles. J. Proteome Res. 9: 5197-5208.

Kearns, D.B., Campbell, B.D., and Shimkets, L.J. (2000). Myxococcus xanthus fibril appendages are essential for excitation by a phospholipid attractant. Proc. Natl. Acad. Sci. USA 97: 11505-11510.

Kim, S.H., Ramaswamy, S., and Downard, J. (1999). Regulated exopolysaccharide production in Myxococcus xanthus. J. Bacteriol. 181: 1496-1507.

Kimura, Y., Ishida, S., Matoba, H., and Okahisa, N. (2004). RppA, a transducer homologue, and MmrA, a multidrug transporter homologue, are involved in the biogenesis and/or assembly of polysaccharide in Myxococcus xanthus. Microbiology 150: 631-639.

Kimura, Y., Kato, T., and Mori, Y. (2012). Function analysis of a bacterial tyrosine kinase, BtkB, in Myxococcus xanthus. FEMS Microbiol. Lett. 336: 45-51.

Kimura, Y., Yamashita, S., Mori, Y., Kitajima, Y., and Takegawa, K. (2011). A Myxococcus xanthus bacterial tyrosine kinase, BtkA, is required for the formation of mature spores. J. Bacteriol. 193: 5853-5857.

Komano, T., Furuichi, T., Teintze, M., Inouye, M., and Inouye, S. (1984). Effects of deletion of the gene for the development-specific protein S on differentiation in Myxococcus xanthus. J. Bacteriol. 158: 1195-1197.

Konovalova, A., Petters, T., and Søgaard-Andersen, L. (2010). Extracellular biology of Myxococcus xanthus. FEMS Microbiol. Rev. 34: 89-106.

Kottel, R.H., Bacon, K., Clutter, D., and White, D. (1975). Coats from Myxococcus xanthus: characterization and synthesis during myxospore differentiation. J. Bacteriol. 124: 550-557.

Lancero, H., Brofft, J.E., Downard, J., Birren, B.W., Nusbaum, C., Naylor, J., Shi, W., and Shimkets, L.J. (2002). Mapping of Myxococcus xanthus social motility dsp mutations to the dif genes. J. Bacteriol. 184: 1462-1465.

Lancero, H.L., Castaneda, S., Caberoy, N.B., Ma, X., Garza, A.G., and Shi, W. (2005). Analysing protein-protein interactions of the Myxococcus xanthus dif signalling pathway using the yeast twohybrid system. Microbiology 151: 1535-1541.

Lee, B., Mann, P., Grover, V., Treuner-Lange, A., Kahnt, J., and Higgs, P.I. (2011). The Myxococcus xanthus spore cuticula protein C is a fragment of FibA, an extracellular metalloprotease produced exclusively in aggregated cells. PLoS ONE 6, https://doi.org/10. 1371/journal.pone.0028968.

Leng, X., Zhu, W., Jin, J., and Mao, X. (2011). Evidence that a chaperoneusher-like pathway of Myxococcus xanthus functions in spore coat formation. Microbiology 157: 1886-1896.

Li, Y., Sun, H., Ma, X., Lu, A., Lux, R., Zusman, D., and Shi, W. (2003). Extracellular polysaccharides mediate pilus retraction during social motility of Myxococcus xanthus. Proc. Natl. Acad. Sci. USA 100: 5443-5448.

Li, Z., Hwang, S., and Bar-Peled, M. (2016). Discovery of a unique extracellular polysaccharide in members of the pathogenic Bacillus that can co-form with spores. J. Biol. Chem. 291: 19051-19067.

Lu, A., Cho, K., Black, W.P., Duan, X.Y., Lux, R., Yang, Z., Kaplan, H.B., Zusman, D.R., and Shi, W. (2005). Exopolysaccharide biosynthesis genes required for social motility in Myxococcus xanthus. Mol. Microbiol. 55: 206-220.

Lukose, V., Walvoort, M.T., and Imperiali, B. (2017). Bacterial phosphoglycosyl transferases: initiators of glycan biosynthesis at the membrane interface. Glycobiology 27: 820-833.

Maclean, L., Perry, M.B., Nossova, L., Kaplan, H., and Vinogradov, E. (2007). The structure of the carbohydrate backbone of the LPS from Myxococcus xanthus strain DK1622. Carbohydr. Res. 342: 2474-2480.

McCleary, W.R., Esmon, B., and Zusman, D.R. (1991). Myxococcus xanthus protein $\mathrm{C}$ is a major spore surface protein. J. Bacteriol. 173: 2141-2145.

Mercier, R. and Mignot, T. (2016). Regulations governing the multicellular lifestyle of Myxococcus xanthus. Curr. Opin. Microbiol. 34: 104-110.

Merroun, M.L., Ben Chekroun, K., Arias, J.M., and González-Muñoz, M.T. (2003). Lanthanum fixation by Myxococcus xanthus: cellular location and extracellular polysaccharide observation. Chemosphere 52: 113-120.

Moak, P.L., Black, W.P., Wallace, R.A., Li, Z., and Yang, Z. (2015). The Hsp70-like StkA functions between T4P and Dif signaling proteins as a negative regulator of exopolysaccharide in Myxococcus xanthus. Peer J. 3: e747.

Mori, Y., Maeda, M., Takegawa, K., and Kimura, Y. (2012). PhpA, a tyrosine phosphatase of Myxococcus xanthus, is involved in the production of exopolysaccharide. Microbiology 158: 2546-2555.

Müller, F.D., Schink, C.W., Hoiczyk, E., Cserti, E., and Higgs, P.I. (2012). Spore formation in Myxococcus xanthus is tied to cytoskeleton functions and polysaccharide spore coat deposition. Mol. Microbiol. 83: 486-505.

Overgaard, M., Wegener-Feldbrugge, S., and Søgaard-Andersen, L. (2006). The orphan response regulator DigR is required for synthesis of extracellular matrix fibrils in Myxococcus xanthus. J. Bacteriol. 188: 4384-4394.

Partridge, J.D. and Harshey, R.M. (2013). Swarming: flexible roaming plans. J. Bacteriol. 195: 909-918.

Pérez-Burgos, M., García-Romero, I., Jung, J., Schander, E., Valvano, M.A., and Søgaard-Andersen, L. (2020a). Characterization of the exopolysaccharide biosynthesis pathway in Myxococcus xanthus. J. Bacteriol. 202 in press, https://doi.org/10.1101/ 2020.06.08.139634.

Pérez-Burgos, M., García-Romero, I., Jung, J., Valvano, M.A., and Søgaard-Andersen, L. (2019). Identification of the lipopolysaccharide 0 -antigen biosynthesis priming enzyme and the 0-antigen ligase in Myxococcus xanthus: critical role of LPS 0 -antigen in motility and development. Mol. Microbiol. 112: 1178-1198.

Pérez-Burgos, M., García-Romero, I., Valvano, M.A., and SøgaardAndersen, L. (2020b). Identification of the Wzx flippase, Wzy polymerase and sugar-modifying enzymes for spore coat polysaccharide biosynthesis in Myxococcus xanthus. Mol. Microbiol. 113: 1189-1208.

Petters, T., Zhang, X., Nesper, J., Treuner-Lange, A., Gomez-Santos, N., Hoppert, M., Jenal, U., and Søgaard-Andersen, L. (2012). The orphan histidine protein kinase SgmT is a c-di-GMP receptor and regulates composition of the extracellular matrix together with the orphan DNA binding response regulator DigR in Myxococcus xanthus. Mol. Microbiol. 84: 147-165. 
Pham, V.D., Shebelut, C.W., Mukherjee, B., and Singer, M. (2005). RasA is required for Myxococcus xanthus development and social motility. J. Bacteriol. 187: 6845-6848.

Raetz, C.R. and Whitfield, C. (2002). Lipopolysaccharide endotoxins. Annu. Rev. Biochem. 71: 635-700.

Ramaswamy, S., Dworkin, M., and Downard, J. (1997). Identification and characterization of Myxococcus xanthus mutants deficient in calcofluor white binding. J. Bacteriol. 179: 2863-2871.

Römling, U., Galperin, M.Y., and Gomelsky, M. (2013). Cyclic di-GMP: the first 25 years of a universal bacterial second messenger. Microbiol. Mol. Biol. Rev. 77: 1-52.

Sabra, W. and Zeng, A.P. (2009). Microbial production of alginates: physiology and process aspects. In: Alginates: biology and applications. Springer, Berlin, Heidelberg, pp. 153-173.

Schumacher, D. and Søgaard-Andersen, L. (2017). Regulation of cell polarity in motility and cell division in Myxococcus xanthus. Annu. Rev. Microbiol. 71: 61-78.

Shi, W., Yang, Z., Sun, H., Lancero, H., and Tong, L. (2000). Phenotypic analyses of frz and dif double mutants of Myxococcus xanthus. FEMS Microbiol. Lett. 192: 211-215.

Shimkets, L.J. (1986a). Correlation of energy-dependent cell cohesion with social motility in Myxococcus xanthus. J. Bacteriol. 166: 837-841.

Shimkets, L.J. (1986b). Role of cell cohesion in Myxococcus xanthus fruiting body formation. J. Bacteriol. 166: 842-848.

Silhavy, T.J., Kahne, D., and Walker, S. (2010). The bacterial cell envelope. Cold Spring Harb. Perspect. Biol. 2: a000414.

Skotnicka, D., Petters, T., Heering, J., Hoppert, M., Kaever, V., and Søgaard-Andersen, L. (2015). c-di-GMP regulates type IV pilidependent-motility in Myxococcus xanthus. J. Bacteriol. 198: 77-90.

Skotnicka, D., Smaldone, G.T., Petters, T., Trampari, E., Liang, J., Kaever, V., Malone, J.G., Singer, M., and Søgaard-Andersen, L. (2016). A minimal threshold of c-di-GMP is essential for fruiting body formation and sporulation in Myxococcus xanthus. PLoS Genet. 12: e1006080.

Sperandeo, P., Martorana, A.M., and Polissi, A. (2019). Lipopolysaccharide biosynthesis and transport to the outer membrane of Gram-negative bacteria. Subcell. Biochem. 92: 9-37.

Sudo, S.Z. and Dworkin, M. (1969). Resistance of vegetative cells and microcysts of Myxococcus xanthus. J. Bacteriol. 98: 883-887.

Sutherland, I.W. and Thomson, S. (1975). Comparison of polysaccharides produced by Myxococcus strains. J. Gen. Microbiol. 89: 124-132.

Thomasson, B., Link, J., Stassinopoulos, A.G., Burke, N., Plamann, L., and Hartzell, P.L. (2002). MglA, a small GTPase, interacts with a tyrosine kinase to control type IV pili-mediated motility and development of Myxococcus xanthus. Mol. Microbiol. 46: 1399-1413.

Valvano, M.A. (2011). Common themes in glycoconjugate assembly using the biogenesis of 0 -antigen lipopolysaccharide as a model system. Biochemistry 76: 729-735.

Wartel, M., Ducret, A., Thutupalli, S., Czerwinski, F., Le Gall, A.V., Mauriello, E.M., Bergam, P., Brun, Y.V., Shaevitz, J., and Mignot, T. (2013). A versatile class of cell surface directional motors gives rise to gliding motility and sporulation in Myxococcus xanthus. PLoS Biol. 11: e1001728.

Weimer, R.M., Creighton, C., Stassinopoulos, A., Youderian, P., and Hartzell, P.L. (1998). A chaperone in the HSP70 family controls production of extracellular fibrils in Myxococcus xanthus. J. Bacteriol. 180: 5357-5368.

Whitfield, C. (2006). Biosynthesis and assembly of capsular polysaccharides in Escherichia coli. Annu. Rev. Biochem. 75: 39-68.

Whitfield, C. and Trent, M.S. (2014). Biosynthesis and export of bacterial lipopolysaccharides. Annu. Rev. Biochem. 83: 99-128.

Whitfield, C., Wear, S.S., and Sande, C. (2020). Assembly of bacterial capsular polysaccharides and exopolysaccharides. Annu. Rev. Microbiol., https://doi.org/10.1146/annurev-micro-011420075607.

Willett, J.W., Tiwari, N., Muller, S., Hummels, K.R., Houtman, J.C., Fuentes, E.J., and Kirby, J.R. (2013). Specificity residues determine binding affinity for two-component signal transduction systems. mBio 4: e00420-00413.

Wolgemuth, C., Hoiczyk, E., Kaiser, D., and Oster, G. (2002). How myxobacteria glide. Curr. Biol. 12: 369-377.

Wu, S.S. and Kaiser, D. (1995). Genetic and functional evidence that Type IV pili are required for social gliding motility in Myxococcus xanthus. Mol. Microbiol. 18: 547-558.

Yan, J., Garza, A.G., Bradley, M.D., and Welch, R.D. (2012). A Clp/ Hsp100 chaperone functions in Myxococcus xanthus sporulation and self-organization. J. Bacteriol. 194: 1689-1696.

Yang, Z., Geng, Y., and Shi, W. (1998a). A DnaK homolog in Myxococcus xanthus is involved in social motility and fruiting body formation. J. Bacteriol. 180: 218-224.

Yang, Z., Geng, Y., Xu, D., Kaplan, H.B., and Shi, W. (1998b). A new set of chemotaxis homologues is essential for Myxococcus xanthus social motility. Mol. Microbiol. 30: 1123-1130.

Yang, Z., Guo, D., Bowden, M.G., Sun, H., Tong, L., Li, Z., Brown, A.E., Kaplan, H.B., and Shi, W. (2000a). The Myxococcus xanthus wbgB gene encodes a glycosyltransferase homologue required for lipopolysaccharide 0-antigen biosynthesis. Arch. Microbiol. 174: 399-405.

Yang, Z. and Li, Z. (2005). Demonstration of interactions among Myxococcus xanthus Dif chemotaxis-like proteins by the yeast two-hybrid system. Arch. Microbiol. 183: 243-252.

Yang, Z., Ma, X., Tong, L., Kaplan, H.B., Shimkets, L.J., and Shi, W. (2000b). Myxococcus xanthus dif genes are required for biogenesis of cell surface fibrils essential for social gliding motility. J. Bacteriol. 182: 5793-5798.

Youderian, P. and Hartzell, P.L. (2006). Transposon insertions of magellan-4 that impair social gliding motility in Myxococcus xanthus. Genetics 172: 1397-1410.

Zhang, Y., Ducret, A., Shaevitz, J., and Mignot, T. (2012). From individual cell motility to collective behaviors: insights from a prokaryote, Myxococcus xanthus. FEMS Microbiol. Rev. 36: 149-164.

Zhou, T., and Nan, B. (2017). Exopolysaccharides promote Myxococcus xanthus social motility by inhibiting cellular reversals. Mol. Microbiol. 103: 729-743. 